\title{
Sperm Chromatin Abnormalities and DNA Damage Predict Assisted Reproductive Outcome
}

Dr. Riffat Bibi ( $\nabla$ riffat.skmc@gmail.com )

Quaid-i-Azam University

Dr. Sarwat Jehan

Quaid-i-Azam University

Dr. Suhail Razak

King Saud bin Abdulaziz University for Health Sciences

Professor Mohammad Eid Hammadeh

Universitätsklinikum des Saarlandes

Dr. Houda Amor

Universitätsklinikum des Saarlandes

\section{Research Article}

Keywords:

Posted Date: February 15th, 2022

DOI: https://doi.org/10.21203/rs.3.rs-1231874/v1

License: (c) (1) This work is licensed under a Creative Commons Attribution 4.0 International License. Read Full License 


\section{Abstract}

This study aimed to evaluate the effect of sperm chromatin quality and DNA fragmentation of spermatozoa on assisted conception outcomes. Patients with male factor infertility (MFI) were grouped into normozoospermic (N), severe male factor (SMF), and mild male factor (MMF) infertility. DNA fragmentation index (DFI) in spermatozoa was analyzed by sperms chromatin dispersion (SCD), sperm chromatin structure assay (SCSA), Acridine Orange testing (AOT), chromatin maturation (CM) including CMA3 to measure protamination and Toluidine Blue (TB) to measure chromatin condensation.

DFI measured by SCD, SCSA, and AOT while CM checked through protamination chromomycin A3 (CMA3) and chromatin condensation TB, were significantly lower in $\mathrm{N}$ compared with infertile groups (MMF and SMF). Embryo aneuploidy was significantly higher in male factor infertile groups (MMF and SMF) compared to N. A positive correlation was observed between fertilization rate (FR) and live birth rate (LBR) with sperm count, motility, vitality, and negative correlation between sperm morphology, sperm DFI, (SCD, SCSA, and AO) sperm CMI (CMA3 and TB). No correlation was observed between embryo aneuploidy and sperm DFI and CMI. Spermatozoa DFI and CMI are associated with low fertilization and live birth rate.

The couples undergoing invitro-fertilization (IVF) or intracytoplasmic injection (ICSI) with male factor infertility; including impaired semen parameters, sperm DNA and chromatin quality could be regarded in the future as an indication and prediction of assisted reproduction techniques (ART), its success.

\section{Introduction}

Infertility is defined as no conception despite one year of unprotected attempts [1]. One initial investigation aimed to find a cause of infertility is the evaluation of semen samples to rule out malefactors; which are attributed to $40-50 \%$ of infertility [2]. Conventional semen analysis includes sperm count, motility, and morphology; however, these parameters do not strictly predict fertilization potential [3]-[5]. Sperm abnormal chromatin and DNA fragmentation assessment are hidden anomalies frequent in infertile men [6]. Subsequently, routine sperm parameters alone do not enable the identification of a substantial proportion of infertile men. Reactive oxygen species (ROS) might also be associated with human infertility and oxidative stress, which is implicated in the etiology of sperm dysfunction and male infertility [7], [8]. In humans, protamines replace approximately $85 \%$ of the histones during the process of spermatogenesis [9], [10].

The presence of damaged DNA could also arise from problems in nuclear remodeling resulting directly from defective protamine deposition during spermiogenesis [10], [11].

The presence of damaged DNA may also result in faulty nuclear remodeling caused by faulty protamine deposition during spermiogenesis. [12]. One of the features of spermatozoa protamines is that they will be concerned with the safety of the genetic code. Incomplete protamination could render the spermatozoa greater at risk of attacks with the aid of using endogenous or exogenous agents, including 
free radicals, mutagens [15], [16], and nucleases [13], [14]. Altered protamine expression reasons multiplied DNA damage that's related to bad semen quality, decreased fertilization capability, poor embryo development, recurrent implantation failure, and a higher miscarriage rate [17], [18].

Sperm contributes haploid genome to embryonic genetic information at the time of fertilization. The frequency of meiotic issues in offspring with numerical and structural chromosomal abnormalities showed a higher prevalence in infertile men compared to the fertile population [19]. Furthermore, infertile men with a higher incidence of DNA fragmentation correlate with poor sperm quality and a higher degree of chromosomal anomalies [17]. Sperm with a higher DNA fragmentation rate and impaired sperm chromatin will increase the prevalence of chromosomal aneuploidy, low pregnancy, and implantation after ICSI [3] [4] [16]. The information obtained so far from the literature available on preimplantation genetic diagnosis for aneuploidy, the severe male factor abnormalities could make contributions to a higher prevalence of chromosomally abnormal embryos even if the female age is under 36 years [20] [21]. Sperm chromatin maturity assessment based on sperm nuclear protein stains (toluidine blue) and chromomycin A3 (CMA3) staining for defective-protamination [22].

Different methods to check sperm chromatin integrity are accomplished by evaluation of sperm chromatin fragmentation index (DFI) utilizing sperm chromatin structure analysis, Sperm Nucleus DNA integrity Kit, terminal transferase dUTP nick-end labeling (TUNEL), the alkaline/acidic comet assay, and sperm chromosomal aberration by in situ hybridization (FISH). Studies showed that SCD and TB are easy and less expensive methods for the dedication of sperm chromatin integrity. Furthermore, the sperm chromatin maturation (CM) is checked by sperm protamine deficiency chromomycin A3 (CMA3) staining and toluidine blue or aniline blue $(A B)$-stain sperm. Although a number of those are onerous and require high-tech machinery, the aim is selection for the methods that may without difficulty and inexpensively examine chromatin status in the routine seminal analysis. As studies are showing that human sperm DNA damage and sperm chromatin maturation can also additionally adversely affect the embryo quality [6], [9], [23], [24]

The present study aimed to evaluate the impact of semen parameters, sperm DNA and chromatin integrity on fertilization rate, cleavage rate, aneuploidy, and live birth rates.

\section{Methods}

\section{Patients}

This prospective study included 700 couples who underwent IVF/ICSI procedure out of which 102 couples were involved in the ICSI/IVF program at Fertility and Genetic services, Islamabad, Pakistan, from April 2016-March 2021. The study protocol was approved by the institutional review board of Quaid-IAzam University. Ethical approval was obtained from the Ethics Committee of SKMC Islamabad Pakistan. All experiments were performed under relevant guidelines and regulations of the SKMC and QAU. Informed consent was obtained from all participants and/or their legal guardians and has been performed per the Declaration of Helsinki. A thorough physical examination was performed, including 
measurement of testicular size to rule out abnormalities of the external genitalia and cryptorchidism; an ultrasonographic examination was performed to rule out varicoceles; an immunobead binding test was performed to rule out the presence of anti-sperm antibodies, and karyotyping was used to rule out the presence of sperm antibodies. A brief medical history, with male body mass index (BMI), male and female age, duration of infertility, primary or secondary infertility, and previous pregnancy loss related data was obtained, primarily by an informal interview with the patient, or from the patient's clinical notes or a self-reported questionnaire.

inki. A thorough physical examination was performed, including measurement of testicular size to rule out abnormalities of the external genitalia and cryptorchidism; an ultrasonographic examination was performed to rule out varicoceles; an immunobead binding test was performed to rule out the presence of anti-sperm antibodies, and karyotyping was used to rule out the presence of sperm antibodies. A brief medical history, with male body mass index (BMI), male and female age, duration of infertility, primary or secondary infertility, and previous pregnancy loss related data was obtained, primarily by an informal interview with the patient, or from the patient's clinical notes or a self-reported questionnaire.

All the participants included in this study were of reproductive age, the range being between 25 and 50 years old. Hormonal profile of male Luteinizing hormone (LH), follicle-stimulating hormone (FSH), and testosterone; and female patient Luteinizing hormone (LH), follicle-stimulating hormone (FSH), and antiMullerian hormone (AMH) were determined by radioimmunoassay (RIA) using commercial kits.

\section{Study design}

Male patients were divided into three groups

normozoospermic $(\mathrm{N}) \mathrm{n}=143$, Moderate male factor (MMF) $\mathrm{n}=280$ \{(Oligozoosperrmia; Os, Asthenozoospermia; As, OligoAsthenozoospermia; $O A s)$ \}, Sever male factor (SMF) $n=277$ $\{($ Teratozoospermia; Ts, Oligoteratozoospermia; OTs, Asthenoteratozoosperrmia; ATs, Oligoasthenoteratozoospermia; OATs\}.

\section{Semen Parameters}

A semen sample was prepared by masturbation on the day of oocyte aspiration and left to liquefy at $37^{\circ} \mathrm{C}$ for 30 minutes before analysis. Each sample was split into two aliquots: one for sperm parameters, vitality, reactive oxygen species, sperm DNA damage, and chromatin abnormalities, and the other for sperm processing to inseminate oocytes through ICSI. According to WHO 2010 standards, sperm counts, motility, and morphology were examined.

\section{Sperm Chromatin Integrity Assessments}

Sperm chromatin is assessed through sperm protamination and DNA fragmentation.

\section{Protamination}


For sperm CM assessment, we used toluidine blue (TB), chromomycin A3 (CMA3), and acridine orange (AO). All chemicals and substances were purchased from Sigma-Aldrich Company (St. Louis, MO, USA). The positive and negative control was definite, separately, with and without acid denaturation ([25].

\section{Staining with toluidine blue (TB)}

Two washed spermatozoa smear, newly preserved with 96 percent ethanol-acetone, were obtained for each sample. After 12 hours, the slides were treated with $0.1 \mathrm{M} \mathrm{HCl}$ at $4{ }^{\circ} \mathrm{C}$ for 5 minutes and then rinsed three times with distilled water for 2 minutes each. After 5-10 minutes, the slides were rinsed with distilled water and coated with Toluidine Blue (TB) solution ( 0.05 percent TB in 50 percent Mcllvain citrate phosphate buffer, $\mathrm{pH} 3.5-4)$. The slides were dehydrated in ethanol baths one after the other $(70,96$, and 100 percent). Finally, the spermatozoa were counted under an optical microscope after the slides were coated and mounted with xylene at room temperature (2-3 minutes). Each sample had 200-500 spermatozoa examined. Spermatozoa stained dark blue were thought to have aberrant chromatin packing. [26], [27].

\section{Chromomycin A3 staining}

The smears were dried and fixed for 10 minutes in Carnoy's solution at $4^{\circ} \mathrm{C}$. For 20 minutes in the darkroom, the slides were stained with $150 \mathrm{ml}$ of CMA3 $(0.25 \mathrm{mg} / \mathrm{ml})$ in Mcllvain buffer. The slides were then rinsed in a buffer before being mounted with buffered glycerol. [18], [28]. Under a fluorescence microscope with a 460-nm filter, at least 100 spermatozoa were counted for each sample; brilliant yellowstained chromomycin-reacted spermatozoa (CMA3+) were judged abnormal, whereas yellowish greenstained (CMA3) were reflected as normal protamination. [27], [29], [30].

\section{Sperm DNA fragmentation index}

For sperm DFI assessment, we used Sperm chromatin dispersion assay (SCD), Sperm Chromatin Structure Assay (SCSA), and Acridine Orange (AO)

\section{Sperm chromatin dispersion assay (SCD)}

The SCD test was measured using a Sperm Nucleus DNA integrity Kit (SCD) (Shenzhen Huakang Biomed Co., Ltd., Shenzhen, China) as reported previously [24] In a summary, the following technique was carried out: A glass coverslip was placed over $60 \mathrm{I}$ of a semen sample in a tube containing fluidized agarose. After 4 minutes at $4^{\circ} \mathrm{C}$, the coverslip was removed. Following that, acid denaturation for 7 minutes and lysis for 20 minutes were performed. The slide was then cleaned for 3 minutes with plenty of distilled water before being dehydrated for 2 minutes in 70 percent, 90 percent, and 100 percent ethanol baths. A total of 500 spermatozoa were manually counted after Wright's staining to evaluate sperm DNA integrity using brightfield microscopy per slide. To determine the degree of sperm DNA integrity, the dispersion of sperm DNA was calculated. It was thought that if the SCD value was less than $30 \%$, it was normal. [31].

\section{Sperm Chromatin Structure Assay (SCSA)}


The SCSA ${ }^{\circledR}$ was conducted following the recommendations of its developer [32], [33] TNE buffer (50 mM Tris $\mathrm{HCl} \mathrm{pH} \mathrm{7.4,} 100 \mathrm{mM} \mathrm{NaCl}, 0.1$ mM EDTA; Merck, Darmstadt, Germany) was used to suspend 2x106 sperm cells to a final volume of $1 \mathrm{ml}$. The SCSA was carried out on a 1/5 aliquot of the spermatozoa suspension, which was mixed with $400 \mathrm{I}$ of acid-detergent solution. After that, 30 seconds of $1.2 \mathrm{~mL}$ acridine orange (AO) staining solution (Sigma, St. Louis, USA) was added. Flow cytometric assessment of the metachromatic shift from green (native DNA) to red (denatured, single-stranded DNA) fluorescence was used to quantify abnormal chromatin structure, which was characterized as a higher susceptibility to acid or heat-induced denaturation in situ. The final product was labeled DFI percent.

\section{Acridine orange test (AOT)}

Acridine orange is a metachromatic fluorescence staining that is used to determine the rate of DNA denaturation [18], [34]. For 10 minutes, newly made $A O(0.19 \mathrm{mg} / \mathrm{ml}$ in Mcllvain phosphate-citrate buffer $[\mathrm{pH}=4]$ ) was used to stain the air-dried fixed smears in Carnoy's solution (methanol/glacial acetic acid, 3:1). Smears were examined using a fluorescent microscope (Zeiss Co., Jena, Germany) with a 460-nm filter on the same day. [30]. At least 100 spermatozoa were evaluated, and the rate of normal (green fluorescent) and abnormal cells (red fluorescent) were described as the percentage.

\section{Egg collection and ovarian stimulation}

After a long protocol with long-acting gonadotrophin-releasing hormone analogs, exogenous gonadotrophins were used to induce multiple follicular growths. At 34-36 hours following delivery of human chorionic gonadotrophin (HCG), oocytes were harvested transvaginally under ultrasound guidance and cultured in human tubal fluid supplemented with $5 \%$ human serum albumin (HSA) in a $5 \%$ $\mathrm{CO} 2$ humidified gas environment at $37^{\circ} \mathrm{C}$. Depending on sperm indices and couples' reproductive histories, oocytes were inseminated using conventional IVF or ICSI. A total of 6,950 oocytes and $5495 \mathrm{MII}$ were collected and inseminated.

\section{Biopsy and embryo culture}

Oocytes were assessed at 16-18 hours after insemination based on the presence of two pronuclei. Individually fertilized oocytes were cultivated and scored 40, 62, 88, and 112 hours after insemination. The number and shape of nuclei and blastomeres were counted, as well as the percentage and kind of fragmentation. [35]. At 62-64 hours after insemination, developing embryos with at least four cells and no more than $40 \%$ fragmentation was individually managed in HEPES-buffered media overlaid with preequilibrated mineral oil for the biopsy procedure. A nucleated blastomere was gently aspirated using a polished glass needle that was placed into the perivitelline space following a 20-meter breach in the zona pellucida. Embryos were properly washed and transferred to blastocyst growth medium after blastomere biopsy. Biopsied samples were shipped to igenomix Dubai where Next-generation sequencing (NGS) analysis for pre-implantation genetic testing for aneuploidy (PGT-A) was performed. Euploidy, haploidy, and polyploidy were defined by the presence of two sets, one set, and three or more sets for the tested chromosomes, respectively; monosomy and trisomy were defined by the presence of an abnormal 
number of copies for one or two chromosomes, respectively; and euploidy, haploidy, and polyploidy were defined by the presence of two sets, one set, and three or more sets for the tested chromosomes.

\section{Statistics analysis}

We used the Statistical Package for the Social Sciences for all statistical analyses (IBM SPSS software, version 20). The distribution of all parameters was normal. The data were reported as the mean standard deviation for the descriptive analysis of the results (SD). Pearson analysis was also utilized to show correlations between the various parameters. ANOVA was used to assess differences in means (P-value 0.05). The Student t-test for pairwise comparisons was used to discover whether means were significantly different from the others. Statistical significance was defined as a P value of less than 0.05 . For those outcomes that were connected to one or more examined parameters, prediction models were built. Binary variant logistic regression was used to look for predictors that were significantly linked to such outcomes. The Hosmer-Lemeshow goodness-of-fit test was used to determine the model's prediction's dependability.

\section{Results}

\section{Demographic parameters}

The results showed that there is no significant difference in demographic characteristics among the patients included in this study.

\section{Semen parameters}

The means sperm concentration, progressive motility, immotile, and abnormal sperm morphology of all the investigated patients were significantly lower in MMF and SMF when compared to control $\mathrm{N}$ group Table 1.

\section{Chromatin integrity}

In addition, the mean value of DFI measured by SCD, SCSA, and AOT staining was and chromatin condensation (protamination) measured by CMA3 staining and TB were significantly deranged in MMF and SMF than N patients. Besides, that vitality measured by HOST levels was increased in SMF compared to the $\mathrm{N}$ (Table 1 ).

\section{ART cycle outcome}

The three groups were homogeneous in retrieved oocytes number, gonadotropins dose, and level of estradiol on HCG day. The fertilization rate was significantly higher in the control compared to MMF and SMF groups. While the live birth rate was slightly higher in $\mathrm{N}$ shown in Table 2.

\section{Preimplantation genetic testing for aneuploidy}


The proportion of embryos carrying chromosomal abnormalities in In-vitro fertilization (IVF) and intracytoplasmic sperm injection (ICSI) embryos in different sperm categories showed in Table 4. Chromosomal abnormalities were significantly higher $(p<0.05)$ in MMF and SMF patients when compared with $\mathrm{N}$ Control. Chromosomal defects types, i.e., monosomy was significantly lower in $\mathrm{N}$ compared to MMF and SMF. The proportion of gonosomal aneuploidy was significantly increased with the severity of the malefactor condition as compared to control patients. Embryos carrying complex abnormalities were more common in MMF patients (Table 3).

\section{Correlation between semen parameters and ART outcome}

There was a positive correlation found between sperm count, motility, and vitality with fertilization rate (FR), and a negative correlation between sperm defective morphology with FR and live birth rate (LBR) was seen. A negative correlation was also seen between immotile sperm and LBR. A positive significant correlation was found between LBR with count and vitality. No correlation was found between embryo aneuploidy and sperm parameters, however, there was a present negative correlation between complex abnormalities with sperm count and a positive correlation with sperm abnormal morphology as depicted in Table 4.

\section{Correlation between Chromatin maturity and ART outcome}

Fertilization rate and live birth rate correlated negatively with sperm chromatin quality; TB, CMA3, and sperm DNA fragmentation; SCD, SCSA, AO, and ROS. A significant correlation was seen between ROS with complex abnormalities. No correlation was found between aneuploidy and sperm CM (CMA3, TB,) and sperm DFI (SCD, SCSA, OA) Table 5.

\section{Estimated coefficients; probability of obtaining Euploid embryos}

Estimated coefficients from the logistic regression model performed that could be used to calculate a probability of obtaining a Euploid embryo $\geq 50 \%$ using Count, HOST, DFI (SCSA, SCD, AOT), and CM (CMA3, TB) as a continuous variable against all couples undergone embryo biopsy and PGT-A (Table 6). The Hosmer-Lemeshow statistic test was used to assess the model's goodness of fit. The difference between the anticipated and observed values were not statistically significant $(x 2=3, p=0.927)$.

\section{Discussion}

Protamines are inserted in the minor groove of double-stranded DNA to create a DNA-Protamine complex during spermatogenesis, progressively replacing nucleoprotein histones [9], [17]. The structure of sperm chromatin is altered when the expression of each kind of sperm-specific nucleoprotein is aberrant, which can lead to male infertility. [9], [22]. In the present study, we found a significant increase in CM levels in moderate (Os, As, TZs) and severe male (OA, NOA, and OAT-S) infertile patients compared with normozoospermic. Our data are in agreement with previous experimental results showing that normal semen samples prevailed in low chromatin and DNA fragmentation alteration [36]. Moreover, the 
percentage of spermatozoa with abnormal CMI and DFI was significantly higher in an infertile group compared with controls as suggested previously[5], [37], [38]. SCD test may be regarded as a potential alternative that allows the better measure of DNA integrity with basic diagnostic laboratories setup [39], [40]. It's less expensive and easier to administer than the more popular SCSA (sperm chromatin structure assay) and TUNEL tests (terminal deoxynucleotidyl transferase-mediated dUTP nick-end labeling) [32], [41].

In addition to the higher level of DFI in the percentage of SCD, SCSA cells in infertile groups compared with normal control, this observation is in agreement with the reports of several studies [24], [32], [33], [42]. Using SCSA, infertile men have a higher percentage of spermatozoa with denatured DNA compared with normal men, suggesting that poor sperm DNA integrity could be one of the causes of infertility [32].

In addition to the importance of sperm DFI assessment, the present study showed that a higher percentage of sperm CM (checked through chromatin condensation and abnormal spermatozoa protamination) influence male fertility, early embryonic development, and recurrent implantation failure and pregnancy outcome [4], [6], [13], [23].

The sperm DFI and CM's functional consequences are still unknown. This is since DNA fragmentation results from apoptosis, recombination anomalies and therefore protamine (P1/P2 ratio) imbalances, oxidative stress, act as intrinsic causative agents or external variables such as temperatures trauma due to cryopreservation. [43].

Reactive oxygen species and oxidative damage are often serious explanations for declining sperm quality; preventing this antioxidants therapy could play a task [44]. Therapeutic correction of this ROS damage has a limited implication. Literature is lacking regarding the mechanism of defect and information is lacking on factors causing decreased sperm fertilization ability.

The result of the present study also showed that in the early stages of in-vitro embryo development there is a difference in a male patient with SMF compare to MMF and $\mathrm{N}$ subjects regarding oocyte fertilization and blastocyst development [45]-[47]. A significantly lower implantation rate was observed in the current study among couples with malefactors (SMF and MMF) compare to $\mathrm{N}$, as suggested by other researchers as well[19], [20], [48]. Similarly, several studies reported a higher frequency of aneuploidy in the case of a severe male factor compared with the normal male population [21], [49]-[51]. SMF (OAs, AZs, and OATs) and MMF (Os, As, TZs) Interestingly in binary logistic regression analysis we found probability to obtaining $\geq 50 \%$ euploid rate in embryos of the infertile couple is depended on sperm quality and determinant of the genetic quality (aneuploidy) of the embryo produced, as Oocyte has the competency to repair paternal chromatin abnormalities but this capability is limited depend on the extent of damage, as evidenced in previously reported study by, [51] which concluded that SMF may contribute to a decreasing number of Euploid blastocysts ( $55 \%$ aneuploidy rate with N, $62 \%$ with oligozoospermia, and $69 \%$ with Obs-AZs), biopsy on day three embryos with in situ hybridizations of few chromosomes was performed. [52] We found higher gonosomal aneuploidy in MMF and SMF as a study [48] conducted 
using NGS on blastomeric biopsies and reported that SMF was associated with a significant increase of sex chromosome abnormalities in embryo compared with subjects with normozoospermic.

In general, conventional semen analysis is insufficient to provide details about the quality and the function of sperm; assessment of the chromatin condensation and DFI is useful tools for assessment of fertilization potential of the spermatozoon. Sperm parameters, sperm DNA damage, and chromatin abnormalities linked with embryo development in patients with male factor infertility [53], [54]. The present study specified that protamine deficiency of chromatin causes abnormal sperm DNA packing raises the susceptibility of sperm DNA to external stresses. Defective sperm chromatin condensation should be considered as sperm abnormality. Higher level of DFI, CMI in male factor infertility, subsequently known to harm embryo kinetics, fertilization, implantation potential, and Live birth rate. [4], [55]-[57]. For this reason, it is strongly suggested to improve spermatogenesis before starting Intracytoplasmic sperm insemination (ICSI) / Invitro fertilization (IVF). During ICSI the chromatin composition and DNA damage of microinjected sperm are unknown. The methods to evaluate sperm DFI (SDF) and CM (CMA3) are less invasive and cheaper compared to SCSA and TUNEL. Hence, it is suggested to do chromatin structure testing before putting the patient through assisted conception procedure and advanced methods for sperm selection with intact chromatin.

An increase in DFI and chromatin condensation did not correlate with embryonic aneuploidy, complex abnormalities, trisomies, and gonosomal aneuploidy this could be either explained by the oocytes' potential to prevent the further development of aneuploidy embryos before the embryonic genome activation or because sperm-derived aneuploidies may result in an early interruption of embryo development. [19], [21], [49]-[51], [58].

The present study is restricted by its sample size. In particular, a higher number of SMF subjects is desirable for future studies, especially to provide more solid evidence investigating the clinical outcomes (obstetrical and perinatal results), for which the present study was underpowered.

SMF with impaired DFI and chromatin condensation causes poor early embryonic competence regarding fertilization rate, developmental potential, and live birth rate. Similarly, we found the embryo euploidy rate and fertilization potential of the obtained embryos are dependent on sperm quality in male factor infertility. Sperm chromatin condensation and DNA integrity may be considered applicable tools to predict ART success.

\section{Abbreviations}

Normozoospermia $=\mathrm{N}$, Severe male factor $=S M F$, Mild male factor $=$ MMF, DNA fragmentation index $=$ DFI, Male factor infertility $=\mathrm{MFI}$, Sperm chromatin dispersion $=S C D$, Sperm chromatin structure assay $=$ SCSA, Acridine Orange testing=AOT, Toluidine Blue =TB, chromomycin A3 = CMA3 Chromatin maturation $=\mathrm{CM}$, Fertilization rate $=\mathrm{FR}$, Live birth rate $=\mathrm{LBR}$, Invitro-fertilization $=$ IVF, Intra cytoplasmic injection $=$ ICSI, Assisted reproduction techniques = ART Reactive oxygen species $=$ ROS, preimplantation genetic screening $=$ PGS, Terminal transferase dUTP nick-end labeling =TUNEL assay, aniline blue (AB)-stain 
sperm chromatin maturation assay $=\mathrm{SCMA}$, Oligozoosperrmia $=0 \mathrm{~s}$, Asthenozoospermia $=\mathrm{As}$, OligoAsthenozoospermia $=$ OAs, Teratozoospermia $=T s$, Oligoteratozoospermia $=0 T s$, Asthenoteratozoosperrmia $=$ ATs Oligoasthenoteratozoospermia= OATs, Obstructive Azoospermia= Obs$A Z s$, human chorionic gonadotrophin $=H C G$, human serum albumin $=H A S$, Next generation sequencing $=$ NGS, pre-implantation genetic testing for aneuploidy $=$ PGT-A, standard deviation $=$ SD, hypo-osmotic swelling test $=$ HOST

\section{Declarations}

\section{Acknowledgments}

Deanship of Scientific Research at King Saud University, We are grateful for its funding of this research through Research Group; Project number 193.

\section{Funding}

The project was partially funded by the Higher Education Commission (HEC) of Pakistan. We are grateful to the Deanship of Scientific Research, King Saud University for its funding of this research through Research Group; Project number 193.

\section{Authors' contributions}

$\mathrm{RB}, \mathrm{SJ}, \mathrm{SR}, \mathrm{MH}, \mathrm{HA}$ contributed significantly to the conceptualization, design, experimentation, data collecting and analysis, and manuscript writing. RB, SJ, SR, MH, HA made a significant contribution to data interpretation and manuscript revision for intellectual content. All authors read and approved the final manuscript.

\section{Ethics approval}

This study makes use of Humans, and the experimental protocol for the use of humans was approved (BAS\#05605) by the ethical board of Quaid-i-Azam University, Islamabad Pakistan. Written consent was taken from participants.

\section{Consent to participate}

This study makes use of humans, and the experimental protocol for the use of humans was approved.

\section{Consent to publication}

Not applicable

\section{Data Availability" / "Availability of data and materials}

All the data are contained in the manuscript. 


\section{Competing interests}

The authors declare that they have no competing interests.

\section{References}

1. N. E. Skakkebæk et al., "Is human fecundity declining?," International Journal of Andrology, vol. 29, no. 1, pp. 2-11, 2006, doi: 10.1111/j.1365-2605.2005.00573.x.

2. T. G. Cooper et al., "World Health Organization reference values for human semen characteristics* $\ddagger$," Human Reproduction Update, vol. 16, no. 3, pp. 231-245, Jan. 2010, doi: 10.1093/humupd/dmp048.

3. M. Cissen et al., "Measuring Sperm DNA Fragmentation and Clinical Outcomes of Medically Assisted Reproduction: A Systematic Review and Meta-Analysis," PLOS ONE, vol. 11, no. 11, p. e0165125, Nov. 2016, doi: 10.1371/journal.pone.0165125.

4. B. Ozmen, N. Koutlaki, M. Youssry, K. Diedrich, and S. Al-Hasani, "DNA damage of human spermatozoa in assisted reproduction: origins, diagnosis, impacts and safety," Reproductive BioMedicine Online, vol. 14, no. 3, pp. 384-395, Jan. 2007, doi: 10.1016/S1472-6483(10)60883-8.

5. A. Wdowiak, S. Bakalczuk, and G. Bakalczuk, "The effect of sperm DNA fragmentation on the dynamics of the embryonic development in intracytoplasmatic sperm injection," Reproductive Biology, vol. 15, no. 2, pp. 94-100, Jun. 2015, doi: 10.1016/j.repbio.2015.03.003.

6. D. Sakkas and J. G. Alvarez, "Sperm DNA fragmentation: mechanisms of origin, impact on reproductive outcome, and analysis," Fertility and Sterility, vol. 93, no. 4, pp. 1027-1036, Mar. 2010, doi: 10.1016/j.fertnstert.2009.10.046.

7. J. Ribas-Maynou, M. Yeste, and A. Salas-Huetos, "The Relationship between Sperm Oxidative Stress Alterations and IVF/ICSI Outcomes: A Systematic Review from Nonhuman Mammals," Biology, vol. 9, no. 7, p. 178, Jul. 2020, doi: 10.3390/biology 9070178 .

8. P. Sabeti et al., "Etiologies of sperm oxidative stress," IJRM, vol. 14, no. 4, pp. 231-240, Apr. 2016, doi: 10.29252/ijrm.14.4.231.

9. C. Niederberger, "Identification and Evaluation of a Novel Sperm Protamine Abnormality in a Population of Infertile Males," Journal of Urology, vol. 174, no. 4 Part 1, pp. 1368-1368, Oct. 2005, doi: 10.1097/S0022-5347(01)68642-7.

10. C. Rathke, W. M. Baarends, S. Awe, and R. Renkawitz-Pohl, "Chromatin dynamics during spermiogenesis," Biochimica et Biophysica Acta (BBA) - Gene Regulatory Mechanisms, vol. 1839, no. 3, pp. 155-168, Mar. 2014, doi: 10.1016/j.bbagrm.2013.08.004.

11. D. Sakkas, E. Seli, G. C. Manicardi, M. Nijs, W. Ombelet, and D. Bizzaro, "The presence of abnormal spermatozoa in the ejaculate: Did apoptosis fail?," Human Fertility, vol. 7, no. 2, pp. 99-103, Jun. 2004, doi: 10.1080/14647270410001720464.

12. M. Spano, E. Seli, D. Bizzaro, G. C. Manicardi, and D. Sakkas, "The significance of sperm nuclear DNA strand breaks on reproductive outcome," Current Opinion in Obstetrics \& Gynecology, vol. 17, no. 3, pp. 255-260, Jun. 2005, doi: 10.1097/01.gco.0000169102.77504.66. 
13. J. T. Carreira et al., "Impaired protamination and sperm DNA damage in a Nellore bull with high percentages of morphological sperm defects in comparison to normospermic bulls," Arq. Bras. Med. Vet. Zootec., vol. 67, no. 2, pp. 417-423, Apr. 2015, doi: 10.1590/1678-7046.

14. M. A. Szczygiel and W. S. Ward, "Combination of Dithiothreitol and Detergent Treatment of Spermatozoa Causes Paternal Chromosomal Damage1," Biology of Reproduction, vol. 67, no. 5, pp. 1532-1537, Nov. 2002, doi: 10.1095/biolreprod.101.002667.

15. J. G. Alvarez et al., "Increased DNA damage in sperm from leukocytospermic semen samples as determined by the sperm chromatin structure assay," Fertility and Sterility, vol. 78, no. 2, pp. 319-329, Aug. 2002, doi: 10.1016/S0015-0282(02)03201-6.

16. D. S. Irvine, J. P. Twigg, E. L. Gordon, N. Fulton, P. A. Milne, and R. J. Aitken, “DNA Integrity in Human Spermatozoa: Relationships With Semen Quality," Journal of Andrology, vol. 21, no. 1, pp. 33-44, 2000, doi: 10.1002/j.1939-4640.2000.tb03273.x.

17. H. Amor, N. Shelko, M. Hamad, A. Zeyad, and M. E. Hammadeh, "An additional marker for sperm DNA quality evaluation in spermatozoa of male partners of couples undergoing assisted reproduction technique (IVF/ICSI): Protamine ratio," Andrologia, vol. 51, Sep. 2019, doi: 10.1111/and.13400.

18. E. Mangoli, M. A. Khalili, A. R. Talebi, S. Ghasemi-Esmailabad, and A. Hosseini, "Is there any correlation between sperm parameters and chromatin quality with embryo morphokinetics in patients with male infertility?," Andrologia, vol. 50, no. 5, Jun. 2018, doi: 10.1111/and.12997.

19. R. Mazzilli et al., "Effect of the male factor on the clinical outcome of intracytoplasmic sperm injection combined with preimplantation aneuploidy testing: observational longitudinal cohort study of 1,219 consecutive cycles," Fertility and Sterility, vol. 108, no. 6, pp. 961-972.e3, Dec. 2017, doi: 10.1016/j.fertnstert.2017.08.033.

20. C. Lei et al., "Conventional ICSI improves the euploid embryo rate in male reciprocal translocation carriers," J Assist Reprod Genet, vol. 38, no. 1, pp. 129-138, Jan. 2021, doi: 10.1007/s10815-02002013-z.

21. K. Pagidas, Y. Ying, and D. Keefe, "Predictive value of preimplantation genetic diagnosis for aneuploidy screening in repeated IVF-ET cycles among women with recurrent implantation failure," $J$ Assist Reprod Genet, vol. 25, no. 2-3, pp. 103-106, Mar. 2008, doi: 10.1007/s10815-008-9200-y.

22. D. Lolis, I. Georgiou, M. Syrrou, K. Zikopoulos, M. Konstantelli, and I. Messinis, "Chromomycin $\mathrm{A}_{3}$ staining as an indicator of protamine deficiency and fertilization," Int J Androl, vol. 19, no. 1, pp. 2327, Feb. 1996, doi: 10.1111/j.1365-2605.1996.tb00429.x.

23. M. Meseguer, R. Santiso, N. Garrido, S. García-Herrero, J. Remohí, and J. L. Fernandez, "Effect of sperm DNA fragmentation on pregnancy outcome depends on oocyte quality," Fertility and Sterility, vol. 95, no. 1, pp. 124-128, Jan. 2011, doi: 10.1016/j.fertnstert.2010.05.055.

24. W.-W. Zheng et al., "Sperm DNA damage has a negative effect on early embryonic development following in vitro fertilization," Asian J Androl, vol. 20, no. 1, p. 75, 2018, doi: 10.4103/aja.aja_19_17.

25. G. C. Manicardi et al., "Presence of Endogenous Nicks in DNA of Ejaculated Human Spermatozoa and its Relationship to Chromomycin A3 Accessibility1," Biology of Reproduction, vol. 52, no. 4, pp. 
864-867, Apr. 1995, doi: 10.1095/biolreprod52.4.864.

26. M. R. Ferrari, S. E. Spirito, S. M. Giuliano, and H. A. Fernández, "Chromatin cytophotometric analysis of abnormal bovine spermatozoa," Andrologia, vol. 30, no. 2, pp. 85-89, Apr. 2009, doi: 10.1111/j.1439-0272.1998.tb01151.x.

27. M. Pourentezari, A. R. Talebi, E. Mangoli, M. Anvari, and M. Rahimipour, "Additional deleterious effects of alcohol consumption on sperm parameters and DNA integrity in diabetic mice," Andrologia, vol. 48, no. 5, pp. 564-569, Jun. 2016, doi: 10.1111/and.12481.

28. P. G. Bianchi, G. C. Manicardi, D. Bizzaro, U. Bianchi, and D. Sakkas, “Effect of Deoxyribonucleic Acid Protamination on Fluorochrome Staining and In Situ Nick-Translation of Murine and Human Mature Spermatozoal," p. 1993, 1993, doi: 1993.

29. E. Mangoli and A. R. Talebi, "Effects of experimentally-induced diabetes on sperm parameters and chromatin quality in mice," . pp, vol. 11, no. 1, p. 8, 2013.

30. A. R. Talebi, E. Mangoli, H. Nahangi, M. Anvari, M. Pourentezari, and I. Halvaei, "Vitamin C attenuates detrimental effects of diabetes mellitus on sperm parameters, chromatin quality and rate of apoptosis in mice," European Journal of Obstetrics \& Gynecology and Reproductive Biology, vol. 181, pp. 32-36, Oct. 2014, doi: 10.1016/j.ejogrb.2014.07.007.

31. P. Sutovsky, "Visualization of Sperm Accessory Structures in the Mammalian Spermatids, Spermatozoa, and Zygotes by Immunofluorescence, Confocal, and Immunoelectron Microscopy," in Germ Cell Protocols, vol. 253, New Jersey: Humana Press, 2004, pp. 059-078. doi: 10.1385/1-59259744-0:059.

32. D. P. Evenson, "The Sperm Chromatin Structure Assay (SCSA®) and other sperm DNA fragmentation tests for evaluation of sperm nuclear DNA integrity as related to fertility," Animal Reproduction Science, vol. 169, pp. 56-75, Jun. 2016, doi: 10.1016/j.anireprosci.2016.01.017.

33. D. P. Evenson, K. L. Larson, and L. K. Jost, "Sperm Chromatin Structure Assay: Its Clinical Use for Detecting Sperm DNA Fragmentation in Male Infertility and Comparisons With Other Techniques," Journal of Andrology, vol. 23, no. 1, pp. 25-43, Jan. 2002, doi: 10.1002/j.1939-4640.2002.tb02599.x.

34. L. Deyebra and R. Oliva, "Rapid Analysis of Mammalian Sperm Nuclear Proteins,” Analytical Biochemistry, vol. 209, no. 1, pp. 201-203, Feb. 1993, doi: 10.1006/abio.1993.1104.

35. M. C. Magli, L. Gianaroli, A. P. Ferraretti, M. Lappi, A. Ruberti, and V. Farfalli, “Embryo morphology and development are dependent on the chromosomal complement," Fertility and Sterility, vol. 87, no. 3, pp. 534-541, Mar. 2007, doi: 10.1016/j.fertnstert.2006.07.1512.

36. C. Chiamchanya, N. Kaewnoonual, P. Visutakul, S. Manochantr, and J. Chaiya, “Comparative study of the effects of three semen preparation media on semen analysis, DNA damage and protamine deficiency, and the correlation between DNA integrity and sperm parameters," Asian J Androl, vol. 12, no. 2, pp. 271-277, Mar. 2010, doi: 10.1038/aja.2009.60.

37. A. De Vos, N. P. Polyzos, G. Verheyen, and H. Tournaye, “Intracytoplasmic morphologically selected sperm injection (IMSI): a critical and evidence-based review," Basic Clin. Androl., vol. 23, no. 1, p. 10, Dec. 2013, doi: 10.1186/2051-4190-23-10. 
38. S. McDowell, B. Kroon, E. Ford, Y. Hook, D. Glujovsky, and A. Yazdani, "Advanced sperm selection techniques for assisted reproduction," Cochrane Database of Systematic Reviews, Oct. 2014, doi: 10.1002/14651858.CD010461.pub2.

39. M. Bungum, L. Bungum, and A. Giwercman, "Sperm chromatin structure assay (SCSA): a tool in diagnosis and treatment of infertility," Asian J Androl, vol. 13, no. 1, pp. 69-75, Jan. 2011, doi: 10.1038/aja.2010.73.

40. C. Niederberger, "Re: Mystery of Idiopathic Male Infertility: Is Oxidative Stress an Actual Risk?," Journal of Urology, vol. 190, no. 5, pp. 1842-1843, Nov. 2013, doi: 10.1016/j.juro.2013.07.075.

41. K. R. Chohan, "Comparison of Chromatin Assays for DNA Fragmentation Evaluation in Human Sperm," Journal of Andrology, vol. 27, no. 1, pp. 53-59, Jan. 2006, doi: 10.2164/jandrol.05068.

42. A. R. Talebi, M. A. Khalili, S. Vahidi, J. Ghasemzadeh, and N. Tabibnejad, "Sperm chromatin condensation, DNA integrity, and apoptosis in men with spinal cord injury," J Spinal Cord Med, vol. 36, no. 2, pp. 140-146, Mar. 2013, doi: 10.1179/2045772312Y.0000000055.

43. C. González-Marín, J. Gosálvez, and R. Roy, "Types, Causes, Detection and Repair of DNA Fragmentation in Animal and Human Sperm Cells," IJMS, vol. 13, no. 12, pp. 14026-14052, Oct. 2012, doi: 10.3390/ijms131114026.

44. S. Ahmadi et al., "Antioxidant supplements and semen parameters: An evidence based review," IJRM, vol. 14 , no. 12 , pp. $729-736$, Dec. 2016, doi: 10.29252/ijrm.14.12.729.

45. D. S. Colaci et al., "Men's body mass index in relation to embryo quality and clinical outcomes in couples undergoing in vitro fertilization," Fertility and Sterility, vol. 98, no. 5, pp. 1193-1199.e1, Nov. 2012, doi: 10.1016/j.fertnstert.2012.07.1102.

46. K. A. Green, G. Patounakis, M. P. Dougherty, M. D. Werner, R. T. Scott, and J. M. Franasiak, "Sperm DNA fragmentation on the day of fertilization is not associated with embryologic or clinical outcomes after IVF/ICSI," J Assist Reprod Genet, vol. 37, no. 1, pp. 71-76, Jan. 2020, doi: 10.1007/s10815-019-01632-5.

47. M. E. Hammadeh, E. Strehler, T. Zeginiadou, P. Rosenbaum, and W. Schmidt, "CHROMATIN DECONDENSATION OF HUMAN SPERM IN VITRO AND ITS RELATION TO FERTILIZATION RATE AFTER ICSI," Archives of Andrology, vol. 47, no. 2, pp. 83-87, Jan. 2001, doi: 10.1080/014850101316901262.

48. A. Coates et al., "Optimal euploid embryo transfer strategy, fresh versus frozen, after preimplantation genetic screening with next generation sequencing: a randomized controlled trial," Fertility and Sterility, vol. 107, no. 3, pp. 723-730.e3, Mar. 2017, doi: 10.1016/j.fertnstert.2016.12.022.

49. I. Gat et al., "Sperm DNA fragmentation index does not correlate with blastocyst aneuploidy or morphological grading," PLOS ONE, vol. 12, no. 6, p. e0179002, Jun. 2017, doi: 10.1371 /journal.pone.0179002.

50. I. Gat et al., "Does the degree of sperm DNA fragmentation affect embryo aneuploidy rate," Fertility and Sterility, vol. 106, no. 3, p. e297, Sep. 2016, doi: 10.1016/j.fertnstert.2016.07.843. 
51. M. Magli, L. Gianaroli, A. Ferraretti, S. Gordts, V. Fredericks, and A. Crippa, “Paternal contribution to aneuploidy in preimplantation embryos," Reproductive BioMedicine Online, vol. 18, no. 4, pp. 536542, Jan. 2009, doi: 10.1016/S1472-6483(10)60131-9.

52. S. Silber, T. Escudero, K. Lenahan, I. Abdelhadi, Z. Kilani, and S. Munné, “Chromosomal abnormalities in embryos derived from testicular sperm extraction," Fertility and Sterility, vol. 79, no. 1, pp. 30-38, Jan. 2003, doi: 10.1016/S0015-0282(02)04407-2.

53. F. G. Iranpour, "Impact of sperm chromatin evaluation on fertilization rate in intracytoplasmic sperm injection," Adv Biomed Res, vol. 3, p. 229, Nov. 2014, doi: 10.4103/2277-9175.145719.

54. S. Marchiani et al., "Chromatin Protamination and Catsper Expression in Spermatozoa Predict Clinical Outcomes after Assisted Reproduction Programs," Sci Rep, vol. 7, no. 1, p. 15122, Dec. 2017, doi: 10.1038/s41598-017-15351-3.

55. V. W. Aoki, L. Liu, and D. T. Carrell, "Identification and evaluation of a novel sperm protamine abnormality in a population of infertile males," Human Reproduction, vol. 20, no. 5, pp. 1298-1306, May 2005, doi: 10.1093/humrep/deh798.

56. E. Mangoli, M. A. Khalili, A. R. Talebi, S. Ghasemi-Esmailabad, and A. Hosseini, "Is there any correlation between sperm parameters and chromatin quality with embryo morphokinetics in patients with male infertility?," Andrologia, vol. 50, no. 5, Jun. 2018, doi: 10.1111/and.12997.

57. R. Oliva, "Protamines and male infertility," Human Reproduction Update, vol. 12, no. 4, pp. 417-435, Aug. 2006, doi: 10.1093/humupd/dml009.

58. M. C. Magli, "Chromosome mosaicism in day 3 aneuploid embryos that develop to morphologically normal blastocysts in vitro," Human Reproduction, vol. 15, no. 8, pp. 1781-1786, Aug. 2000, doi: 10.1093/humrep/15.8.1781.

\section{Tables}

TABLE 1 Summary of semen analysis and sperm chromatin assay results in 700 men undergoing ART treatment 


\begin{tabular}{|c|c|c|c|c|}
\hline Variable & $\begin{array}{l}N \\
(143)\end{array}$ & $\begin{array}{l}\text { MMF } \\
(280)\end{array}$ & $\begin{array}{l}\text { SMF } \\
(277)\end{array}$ & $\begin{array}{l}\text { TOTAL } \\
(700)\end{array}$ \\
\hline $\begin{array}{l}\text { Mean age men in the } \\
\text { year }\end{array}$ & $37.93 \pm 8.09$ & $39.10 \pm 7.6$ & $39.1 \pm 7.4$ & 0.524 \\
\hline Count $\times 10^{6}$ & $\begin{array}{l}91 \pm 42(22- \\
225)\end{array}$ & $\begin{array}{l}56.7 \pm 54(0.08- \\
247)^{\star}\end{array}$ & $\begin{array}{l}43 \pm 58(0.05- \\
313)^{\star}\end{array}$ & $\begin{array}{l}58 \pm 56(0.5- \\
313)\end{array}$ \\
\hline$\%$ Progressive Motility & $\begin{array}{l}58 \pm 11(20- \\
80)\end{array}$ & $37 \pm 9(2-80)$ * & $17 \pm 19(0-84)^{*}$ & $33 \pm 23(0-84)$ \\
\hline$\%$ Immotile & $23 \pm 13(1-75)$ & $54 \pm 23(10-95)$ * & $57 \pm 33(0-100)^{\star}$ & $49 \pm 29(0-100)$ \\
\hline \% Ab Morphology & $93 \pm 3(80-93)$ & $95 \pm 1.8(91-99)^{\star}$ & $97 \pm 0.8(92-99) *$ & $95 \pm 2.5(80-99)$ \\
\hline TB & $18 \pm 6(7-43)$ & $19 \pm 9(6-42)$ & $42 \pm 8(9-73)^{\star}$ & $28 \pm 14(6-73)$ \\
\hline CMA3 & $18 \pm 6(10-37)$ & $24 \pm 8(10-49)$ * & $39 \pm 8(13-69)$ * & $29 \pm 11(10-69)$ \\
\hline$S C D$ & $14 \pm 4(9-36)$ & $21 \pm 6(9-40)$ * & $32 \pm 8(2-66) *$ & $24 \pm 9.5(2-66)$ \\
\hline SCSA & $15 \pm 5(8-39)$ & $22 \pm 8(9-42) *$ & $36 \pm 9(3-45) *$ & $26 \pm 12(3-45)$ \\
\hline AOT & $14 \pm 12(3-68)$ & $17 \pm 9(7-35)$ * & $54 \pm 12(6-77) \star$ & $31 \pm 21(3-77)$ \\
\hline HOST & $\begin{array}{l}88 \pm 11(49- \\
96)\end{array}$ & $73 \pm 11(17-88) \star$ & $36 \pm 8(0-92) *$ & $69 \pm 21(0-96)$ \\
\hline
\end{tabular}

Values represent Mean $\pm S E M ; N$, normozoospermic; MMF, Moderate Male Factor; SMF, Sever Male Factor; The data showed by mean \pm SD (min-max). TB, toluidine blue; CMA3, chromomycin A3, SCD, sperm chromatin dispersion, SCSA (Sperm Chromatin Structure Assay), AOT, acridine orange test; HOST, hypo-osmotic swelling test.

$P<0.001 * \star \star, P<0.01 * \star, P<0.05 *$

Table 2 Summary of embryologic outcomes 


\begin{tabular}{|lllll|}
\hline Variables & N & MMF & SMF & P- \\
\hline No of cycle & 143 & 280 & 277 & \\
\hline $\begin{array}{l}\text { Mean no of retrieved oocytes } \\
(+S D)\end{array}$ & $10.23+7.3$ & $10.0+6.9$ & $10.1+6.59$ & 0.458 \\
\hline No of transfer cycles & 102 & 172 & 189 & \\
\hline Fertilization rate $(\%)$ & $70 / 284(24.16)$ & $84 / 467(17.98)^{*}$ & $103 / 676(15.23)^{*}$ & $.01^{*}$ \\
\hline Live birth rate $(\%)$ & $(24 / 70) 34$ & $(32 / 84) 27^{*}$ & $(32 / 103) 31$ & $.024^{*}$ \\
\hline
\end{tabular}

Values represent Mean \pm SEM; N, normozoospermic; MMF, Moderate Male Factor; SMF, Sever Male Factor. $\mathrm{P}<0.001 * \star \star, P<0.01 * \star, P<0.05^{\star}$

Table 3 Pre-implantation genetic screening (PGS) analysis of embryos in different categories of infertile male patients.

\begin{tabular}{|c|c|c|c|c|c|}
\hline & $\begin{array}{l}N \\
(n: 152)\end{array}$ & $\begin{array}{l}\text { MMF } \\
(n: 144)\end{array}$ & $\begin{array}{l}\text { SMF } \\
(n: 148)\end{array}$ & Total & p-Value \\
\hline Aneuploidy \% & 50.60 & $70.90 *$ & $65.29 *$ & 62.84 & $.007 *$ \\
\hline Gonosomal aneuploidy \% & 19.35 & $22.35^{\star}$ & $38.21 *$ & 29.25 & $.002 *$ \\
\hline Monosomies \% & 13.44 & $31.20 *$ & $23.26^{\star}$ & 22.47 & $.001 *$ \\
\hline Trisomies (\%) & 18.79 & 27.32 & 26.57 & 24.57 & .158 \\
\hline Haploidy and polyploidy (\%) & 6.47 & 7.48 & $15.76^{\star}$ & 11.08 & $.01^{\star}$ \\
\hline Normal XY & 18.85 & 14.32 & 13.32 & 15.14 & .119 \\
\hline Normal XX & 33.59 & 26.69 & 23.79 & 27.20 & .251 \\
\hline Complex abnormalities (\%) & 29.53 & 47.71 & 41.87 & 40.87 & .616 \\
\hline No information & 16.68 & 19.96 & 18.77 & 18.49 & .233 \\
\hline
\end{tabular}

Values represent Mean \pm SEM; N, normozoospermic; MMF, Moderate Male Factor; SMF, Sever Male Facto; PGT-A, Preimplantation Genetic Testing for Aneuploidies.

$P<0.001 * \star \star, P<0.01 * \star, P<0.05 *$ 
Table 4 Correlation between sperm parameters with clinical outcomes results in the studied group

\begin{tabular}{|llllll|}
\hline & $\begin{array}{l}\text { Concentration } x \\
10^{6}\end{array}$ & $\begin{array}{l}\text { \% Progressive } \\
\text { motility }\end{array}$ & $\begin{array}{l}\text { \% } \\
\text { Immotile }\end{array}$ & $\begin{array}{l}\text { \% Ab } \\
\text { morphology }\end{array}$ & Vitality \\
\hline FR & $r=.223^{* *}$ & $r=.078^{*}$ & $r=-.068$ & $r=-.098^{* *}$ & $r=.220^{* *}$ \\
& $p=.000$ & $p=.028$ & $p=.051$ & $p=.008$ & $p=.000$ \\
\hline CR & $r=-.020$ & $r=.028$ & $r=.027$ & $r=.005$ & $r=-.050$ \\
& $p=.314$ & $p=.252$ & $p=.257$ & $p=.457$ & $p=.115$ \\
LBR & $r=.320^{* *}$ & $r=.066$ & $r=-.210^{*}$ & $r=-.277^{* *}$ & $r=.282^{* *}$ \\
\hline aneuploidy \% & $p=.003$ & $p=.287$ & $p=.035$ & $p=.008$ & $p=.007$ \\
\hline Complex & $r=-.077$ & $r=.140$ & $r=-.047$ & $r=-.116$ & $r=.001$ \\
abnormalities (\%) & $p=.220$ & $p=.080$ & $p=.321$ & $p=.124$ & $p=.495$ \\
\hline No information & $r=-.214^{*}$ & $r=-.088$ & $r=-.170$ & $r=.170^{*}$ & $r=-.013$ \\
& $p=.016$ & $p=.190$ & $p=.045$ & $p=.044$ & $p=.449$ \\
\hline & $r=-.108$ & $r=.010$ & $r=-.126$ & $r=.078$ & $r=-.029$ \\
& $p=.139$ & $p=.459$ & $p=.104$ & $p=.220$ & $p=.388$ \\
\hline
\end{tabular}

Ab morphology, abnormal morphology; FR (fertilization rate); CR, Clinical pregnancy rate; LBR (live birth rate).

$P<0.001 * \star *, P<0.01 * *, P<0.05^{*}$

Table 5 Correlation between sperm chromatin tests with clinical outcomes in the studied group 


\begin{tabular}{|lllllll|}
\hline & TB & CMA3 & SCD & SCSA & AO & ROS \\
\hline FR & $r=-.140^{* *}$ & $r=-.091^{*}$ & $r=-.131^{* *}$ & $r=-.098^{* *}$ & $r=-.181^{* *}$ & $r=-.146^{* *}$ \\
& $p=.000$ & $p=.014$ & $p=.001$ & $p=.009$ & $p=.000$ & $p=.000$ \\
\hline LBR & $r=-.329^{* *}$ & $r=-.298^{* *}$ & $r=-.194^{*}$ & $r=-.292^{* *}$ & $r=-.321^{* *}$ & $r=-.273^{* *}$ \\
& $p=.002$ & $p=.005$ & $p=.048$ & $p=.006$ & $p=.003$ & $p=.010$ \\
\hline aneuploidy \% & $r=-.024$ & $r=-.008$ & $r=-.054$ & $r=-.014$ & $r=-.080$ & $r=.058$ \\
& $p=.406$ & $p=.469$ & $p=.295$ & $p=.444$ & $p=.211$ & $p=.287$ \\
\hline Complex abnormalities (\%) & $r=.033$ & $r=.067$ & $r=.154$ & $r=.009$ & $r=.128$ & $r=.173^{*}$ \\
& $p=.370$ & $p=.251$ & $p=.061$ & $p=.466$ & $p=.100$ & $p=.044$ \\
& $r=.056$ & $r=.098$ & $r=.186^{*}$ & $r=.072$ & $r=.060$ & $r=.124$ \\
& $p=.290$ & $p=.164$ & $p=.031$ & $p=.236$ & $p=.274$ & $p=.112$ \\
\hline
\end{tabular}

FR (fertilization rate); LBR (live birth rate); TB, toluidine blue; CMA3, chromomycin A3, SCD, sperm chromatin dispersion, SCSA (Sperm Chromatin Structure Assay), AOT, acridine orange test; ROS, Reactive Oxygen Species.

$P<0.001 * \star \star, P<0.01 * \star, P<0.05 *$

Table 6 Binary logistic regression model to predict euploid $\geq 50 \%$ of embryo's biopsied for PGT-A.

\begin{tabular}{|lcccccc|}
\hline & B & S.E. & & p-value & \multicolumn{2}{c|}{ 95\% C.I.for EXP(B) } \\
\cline { 5 - 7 } & & & & Lower & Upper \\
\hline TB & -.080 & .042 & .056 & .851 & 1.002 \\
CMA3 & .144 & .050 & .004 & 1.048 & 1.273 \\
SCD & -.094 & .043 & .030 & .836 & .991 \\
SCSA & -.028 & .037 & .456 & .905 & 1.046 \\
HOST & .023 & .016 & .158 & .991 & 1.056 \\
\hline Concentration $\times 10^{6}$ & -.015 & .007 & .034 & .972 & .999 \\
\hline Constant & -.711 & 1.450 & .624 & & \\
\hline
\end{tabular}


SE: standard error; Cl: confidence interval; P-value probability; TB, toluidine blue; CMA3, chromomycin A3, SCD, sperm chromatin dispersion, SCSA (Sperm Chromatin Structure Assay); HOST, hypo-osmotic swelling test.

$P<0.001 * \star \star, P<0.01 * *, P<0.05 *$ 